\title{
Design and Implementation of Elevator Internet of Things Security Control System based on Cloud Computing
}

\author{
Juanjuan $\mathrm{Li}^{1, \mathrm{a}}$ \\ ${ }^{1}$ Shaanxi Institute of International Trade\&Commerce, Xi'an, Shaanxi, 712046 \\ ${ }^{\mathrm{a}}$ email
}

Keywords: Cloud Computing, Security System, Internet of Things

\begin{abstract}
In order to overcome the shortcomings of the traditional elevator management and maintenance methods, this paper proposes to establish an elevator safety monitoring system based on the Internet of Things technology. The security monitoring system design framework of the elevator consists of the perception layer, convergence layer and transmission application layer. The system hardware adopts the modular design method to design the central control module, the data acquisition module, the GPRS module, the RF processing module and the power module respectively. The system software The basic information module, maintenance information module, monitoring information module and data analysis module are designed, and the elevator safety monitoring and management system is established. The elevator safety monitoring system based on Internet of Things realizes the automation and informationization of elevator safety monitoring and management, and has wide social and economic benefits for its application and promotion.
\end{abstract}

\section{Introduction}

As a kind of public transport, elevator has been widely used in high-rise residential buildings, large shopping malls, office buildings and other public places. For a long time, the casualties caused by elevator accidents have occurred frequently in our country. The incidence and severity of accidents are much higher than those in developed countries and regions. As a special equipment closely related to people's life safety, elevator safety Operation is getting more and more attention from the government and society. As of 2013, the number of elevators in our country has exceeded 2 million units and has been increasing at an annual rate of about 20\%. However, the existing inspectors have been unable to meet the needs of elevator troubleshooting, regular maintenance, failure of safety components, missing inspection of elevators, How to ensure the safe use of elevators and minimize losses so as to give the people a safe living environment and become a city manager Top priority to consider. The traditional way of elevator management and maintenance has been far from being able to meet the needs of the current situation. It is imperative to apply the emerging Internet of Things technology to elevator monitoring and realize the automation of elevator safety monitoring and management. The Internet of Things (IoT) is based on the Internet of the computer and utilizes a technology such as RFID, advanced sensors and wireless data communication to construct a network covering all kinds of objects in the world, in which objects can communicate with each other without human Intervention. Its essence is to use radio frequency automatic identification technology to realize the automatic identification of objects and the interconnection and sharing of information through the computer Internet. Managers can realize intelligent identification, location, tracking, monitoring and management of objects through computers or mobile phones. The elevator safety monitoring system based on the Internet of Things technology collects various monitoring parameters in real time through the sensor network and transmits the monitoring parameters to the monitoring platform through a wireless or wired network so as to realize intelligent management and maintenance of the elevator. Under this management mode, the running status of the elevator can be monitored in real time, and the status information can be sent to the elevator safety management and monitoring center through the network to timely realize the information exchange and processing between people and the elevator. The establishment of elevator safety monitoring system on the one hand real-time monitoring of elevator status, early 
warning and alarm of the fault, reduce the incidence of elevator failure and improve repair speed elevator fault; the other side of the escalator can provide accurate and timely maintenance of technical support.

\section{elevator safety monitoring system components}

Elevator safety monitoring system is mainly for real-time monitoring of a number of characteristic parameters that affect the safe operation of the elevator, such as: traction, rope tension, distance, speed, balance coefficient, acceleration and deceleration, car quality, safety gear characteristics, elevator doors closed Kinetic energy and speed, pressure of hydraulic elevator, electromagnetic anti-interference ability and temperature control ability of elevator control cabinet and other parameters. These monitoring parameters can be transmitted to the computer monitoring and management system through wireless transmission technology. The computer monitoring and management system analyzes the actual operation status of the elevator And maintain the situation of a unified management.

The sensing layer is composed of various sensors installed in various parts of the periphery of the elevator. The design principle is that the sensor signals and the internal running signals of the elevator are independent from each other, so as to avoid mutual influence. The sensor signals real-time monitor the physical and environmental conditions of each position of the elevator, Elevator safety features parameters, etc., they can be transmitted to the convergence layer within 24 hours of uninterrupted transmission of information. In the elevator site will be arranged RFID test points, check points to save the elevator installation, maintenance and inspection information, the information for the timely maintenance of the elevator to provide technical basis. The core of the convergence layer is the aggregation sub-station, the aggregation sub-station organizes all kinds of information from the perception layer, and conducts a preliminary analysis of the data according to the requirements. The analysis results are differentiated, and the processing results are graded according to the elevator operation conditions. In case of serious personal injury, alarm information should be sent out immediately. In other cases, it is stored locally and sent to the application layer for further processing through the transport layer. The transmission application layer mainly transmits data to the application layer platform through General Packet Radio Service (GPRS) technology and uses the Internet to realize the management and maintenance of all related parties. The application layer platform is a management system for safe operation monitoring, failure analysis and maintenance records of elevators. One of the tasks of the application layer platform is to receive the real-time data transmitted from each substation and analyze the data of elevator operation records over a certain time period to fully understand Real-time elevator operating status and fault distribution. The potential failures and failures have been timely and accurate delivery to the relevant units and individuals, and for property management, government regulatory agencies and maintenance units to provide a query platform. The second task is to truthfully record the elevator installation, renovation, maintenance, repair, inspection and other operational situations, through the wireless transmission technology and on-site RFID store information consistent for the elevator maintenance query, issue early warning information to provide technical support. The elevator safety monitoring system is mainly composed of hardware and software. The hardware part is mainly composed of the site aggregation. It includes the monitoring terminal and the RFID tag design. The monitoring terminal is a central control system which is composed of an ARM controller. The RFID tag uses the Internet of Things Unified coding system Ecode coding, which is a new coding system developed by China Article Coding Center on the basis of a comprehensive international mature coding system based on China's national conditions. It provides a complete coding, identification and resolution solution. The software part is divided into monitoring terminal software and monitoring management system software design, hardware is the physical basis, the software is the soul of the system. In this paper, Hangzhou Xizi Otis Elevator Co., Ltd. as a cooperative unit to elevator traction, rope tension, safety features and elevator door closing force as the main safety monitoring parameters, the use of wireless transmission technology to achieve the communication between signals on the elevator safety monitoring System design of the main 
hardware and software research.

Elevator safety monitoring system consists of data acquisition and audio and video monitoring system 2 parts. The data acquisition device is installed in the engine room, connected with each sensor and safety circuit of the elevator, and transmits the operation status and safety information of the elevator car in the scene to the audio and video monitoring system. At the same time, the audio and video monitoring system installed on the top of the car always monitors the car's image and sound signals and transmits all the information to the monitoring center through the 3G module. Real-time audio and video collection is achieved through humanized interactive software. Alarm, sleepy rescue, daily management, quality assessment, hidden dangers and other functions. The elevator data collector is installed in the engine room to monitor the running status of the elevator. It mainly includes switch door command, up and down arrow, car door lock and other information. The safety information includes upper limit, lower limit, brake coil, maintenance signal, Clip signal and so on. The main function of the data acquisition device is to connect the current sensor to the signal acquisition control end of the elevator computer room to obtain the analog signal of the sensor in the original circuit of the elevator and convert it into a corresponding digital signal and send it to the processor for data collection and aggregation. And then through the 485 bus will handle the data through serial communication to the audio and video monitoring system. Figure 2 for the data collector. To ensure functionality and cost-effective premise, the design choice to the core of the microcontroller, the microprocessor used ST (ST) to produce high-end 32-bit microcontroller STM32, which greatly simplify the peripheral circuit Design, improve product reliability. In the design of hardware anti-jamming, the principle of suppressing the interference source, cutting off the interference propagation path and improving the anti-jamming performance of the sensitive device has been determined, and satisfactory results have been obtained from these three aspects.

\section{Monitoring center platform software design framework}

Elevator safety monitoring system software is designed to build an Ethernet elevator information database, automatically collect real-time information of each elevator, the establishment of record logs, send e-mail and text messages to the maintenance department in case of failure, as long as a small number of safety monitoring personnel will be able to achieve Safety control of all elevators in the entire area. The user management menu includes functions of adding users, editing users, switching users and exiting the system submenu. The main menu is used for functions such as addition, deletion, editing, and switching of user information. The site management menu includes gateway address management, site information management, elevator information management, Data setting and automatic acquisition setting submenu, mainly used for data information management and setting; record management menu contains fault record, overhaul record, lock ladder record and driver record submenu, mainly used for record class log maintenance and management; database Management menu contains backup database, restore database, empty the database, initialize the database, mail log submenu, mainly used for database management; Help menu contains help information, PC software version and monitoring module software version, mainly used to help the function and Hardware and software version information.

The elevator safety monitoring system software is a system software of B / S architecture written by Microsoft $\mathrm{C} \#$ and has such functions as map positioning, real-time monitoring, data drawing, list display, data storage, historical inquiry and early warning information. The data analysis software of the data processing center has the functions of real-time establishing the mathematical model for the received signal, calculating the required data through the optimization algorithm, initially evaluating the test result to judge the operating status of the equipment and the safety level of the car, and generating the charts, Issued a test report and other functions.

\section{Conclusion}

The completed elevator safety monitoring system based on the Internet of Things technology can 
monitor the real running status of the elevator in real time, and can timely provide the reminding information for the fault and the alarm information that appear, and can classify the alarm information according to the severity; the system adopts GPRS wireless Transmission technology, no need to arrange a lot of network lines in the field, the installation and construction is simple, easy to maintain, wide adaptability, suitable for any type of elevator installation; in monitoring and management system, can automatically record the elevator operating status data, based on data analysis results Information on the failure has occurred, can automatically analyze the cause of the malfunction, to help maintenance personnel to promptly solve the problem; competent units, property management units and security units can log in through the network for easy supervision and management. The use of Internet of Things technology to achieve the city elevator running status monitoring and management of the elevator multi-coordinated management of the city elevator operation and management provides a scientific and effective method. The monitoring and management system based on Internet of Things technology completes related management work when no one is in the field, which brings great convenience to the elevator management work. On the one hand, it improves work efficiency and management effect and reduces the labor intensity of the maintenance staff, On the other hand to ensure the safe operation of the elevator to the greatest extent, with a wide range of social and economic benefits.

\section{Acknowledgement}

Project: Research on wireless energy saving and control system of street lamp based on ZigBee technology

Project No.:2017JM6111

Basic research program of Natural Science in Shaanxi Province,2017JM6111

\section{References}

[1] Zhang Min, Chen Chunjun, Huang Hailian. Design of elevator real-time monitoring system based on internet of things [J]. China Measurement \& Test, 2012,38 (1): 101-105.

[2] Zhang Demin, Li Lian. Design of monitoring and controlsystem for elevator internet based on CANBus [J]. Auto-mation \& Instrumentation, 2012 (8): 38-41.

[3] Li Zhenxiang, Li Guo, Li Dexing. Design and implemention of intelligent security management system of RFID based on ARM [J]. Computer Engineering and Design, 2010 (12): 2745-2748.

[4] Jordan R, Abdallan T. Wireless communications and net-working: An overview [J]. IEEE Antenna's and Propaga tion Magazine, 2002, 44 (1): 185-193.

[5] Zhang Demin, Yu Hang, Liu Hongjin. Design of elevator security control system based on smart card [J]. Journal of Tianjin University of Technolgy, 2012, 28 (1): 54-59. 